\title{
Gamma-ray Spectrometers using Superconducting Transition Edge Sensors with External Active Feedback Bias
}

D. T. Chow, M. L. van den Berg, A. Loshak, M. Frank, T. W. Barbee, Jr., and S. E. Labov

This article was submitted to Applied Superconductivity Conference 2000

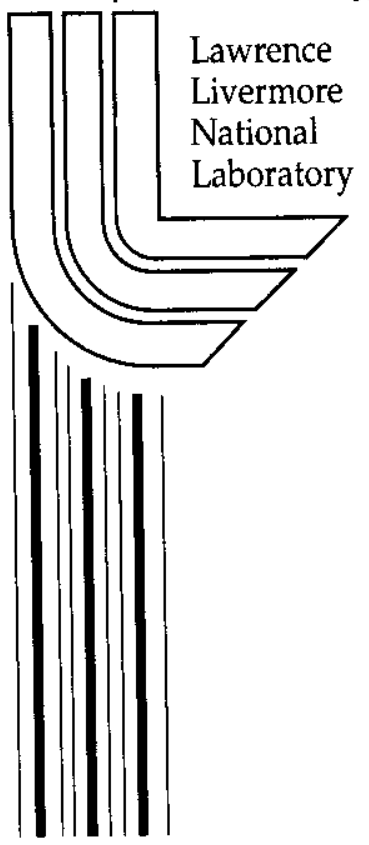

Virginia Beach, VA

September 17-22, 2000

September 22, 2000 


\section{DISCLAIMER}

This document was prepared as an account of work sponsored by an agency of the United States Government. Neither the United States Government nor the University of California nor any of their employees, makes any warranty, express or implied, or assumes any legal liability or responsibility for the accuracy, completeness, or usefulness of any information, apparatus, product, or process disclosed, or represents that its use would not infringe privately owned rights. Reference herein to any specific commercial product, process, or service by trade name, trademark, manufacturer, or otherwise, does not necessarily constitute or imply its endorsement, recommendation, or favoring by the United States Government or the University of California. The views and opinions of authors expressed herein do not necessarily state or reflect those of the United States Government or the University of California, and shall not be used for advertising or product endorsement purposes.

This is a preprint of a paper intended for publication in a journal or proceedings. Since changes may be made before publication, this preprint is made available with the understanding that it will not be cited or reproduced without the permission of the author.

This report has been reproduced directly from the best available copy.

Available to DOE and DOE contractors from the

Office of Scientific and Technical Information

P.O. Box 62, Oak Ridge, TN 37831

Prices available from (423) 576-8401

http:/ /apollo.osti.gov/bridge/

Available to the public from the National Technical Information Service

U.S. Department of Commerce 5285 Port Royal Rd., Springfield, VA 22161

http://www.ntis.gov/

OR

Lawrence Livermore National Laboratory

Technical Information Department's Digital Library

http://www.llnl.gov/tid/Library.html 


\title{
Gamma-ray Spectrometers using Superconducting Transition Edge Sensors with External Active Feedback Bias
}

\author{
D. T. Chow, M. L. van den Berg, A. Loshak, M. Frank, T. W. Barbee, Jr., S. E. Labov
}

\begin{abstract}
We are developing $x$-ray and gamma-ray spectrometers with high absorption efficiency and high energyresolution for $x$-ray and gamma-ray spectroscopy. They are microcalorimeters consisting of a bulk $\mathrm{Sn}$ absorber coupled to a $\mathrm{Mo} / \mathrm{Cu}$ multilayer superconducting transition edge sensor (TES). We have measured an energy resolution of $70 \mathrm{eV}$ FWHM for 60 keV incident gamma-rays using electrothermal feedback. We have also operated these microcalorimeters with an external active feedback bias to linearize the detector response, improve the count rate performance, and extend the detection energy range. We present $x$-ray and gamma-ray results operation of this detector design in both bias modes.
\end{abstract}

Index Terms_Cryogenic detectors, microcalorimeters, transition-edge sensors, bulk absorbers, active feedback bias.

\section{INTRODUCTION}

The Lawrence Livermore National Laboratory is interested in the development of high energy-resolution, high efficiency gamma-ray spectrometers for nuclear non-proliferation and arms control programs [1]. These gamma-ray detectors can be used to identify isotopic compositions of nuclear materials intended for different uses.

In cryogenic microcalorimeters, the energy resolution is limited by thermal phonon fluctuations [2]. Phonon noise is independent of energy and is given by $\Delta E \approx 2.35 \sqrt{k T^{2} C}$, where $k$ is Boltzman's constant, $T$ is the operating temperature, and $C$ is the heat capacity of the detector. Superconducting materials operate at low temperatures and have small heat capacities, making them ideal for this application [3][4]. In our detector design, the typical phonon noise is $\sim 20 \mathrm{eV}$ at the operating temperature of $0.1 \mathrm{~K}$.

\section{DETECTOR DESIGN AND EXPERIMENTAL SETUP}

A microcalorimeter consists of an absorber, a sensitive

D. T. Chow is with the Lawrence Livermore National Laboratory, Livermore, CA 94550 USA and Univ. of Califomia, Davis, Dept. of Appl. Science, Livermore, CA 94550 USA (telephone: $925-422-4525$, e-mail: dchow@llnl.gov).

M. L. van den Berg, A. Loshak, M. Frank, T. W. Barbee, Jr., and S. E. Labov are with the Lawrence Livermore National Laboratory, Livernore, CA 94550 USA.

This work was performed under the auspices of the U.S. Department of Energy by University of California Lawrence Livermore National Laboratory under contracl No. W-7405-Eng-48, with support from NASA Constellation$X$ Technology Development Program under interagency agreement S10256G and the NASA High Energy Astrophysics Supporting Research and Technology Program under interagency agreement W-19.121. thermometer, and a weak thermal link to a cold bath. In our detector design, we use bulk polycrystalline $S n$ as the absorber. Sn has been demonstrated to be a good absorber for $x$-ray and gamma-ray microcalorimeters [5][6][7]. The dimensions of the $S n$ absorber are $1 \mathrm{~mm} \times 1 \mathrm{~mm} \times 0.25 \mathrm{~mm}$, which has a $70 \%$ absorption efficiency at $60 \mathrm{keV}$. We use a $\mathrm{Mo} / \mathrm{Cu}$ multilayer transition edge sensor (TES) with critical temperature $\mathrm{T}_{\mathrm{C}} \sim 0.1 \mathrm{~K}$ as the thermometer [1]. A TES is a superconducting film with a very narrow transition between zero resistance and normal resistance such that small changes in temperature cause large changes in resistance. A silicon nitride (SiN) membrane substrate serves as the weak thermal link to the cold bath with typical thermal conductivity $\mathrm{G}_{\mathrm{SiN}} \sim$ $0.25 \mathrm{nW} / \mathrm{K}$ at a bath temperature of $70 \mathrm{mK}$ [8]. The bulk $\mathrm{Sn}$ is strongly coupled to the TES with a small amount of Stycast epoxy. The dimensions of the epoxy are typically $100 \mu \mathrm{m}$ diameter and $25 \mu \mathrm{m}$ thick, with mass $\sim 0.5 \mu \mathrm{g}$. The combined boundary and bulk thermal conductivity of the epoxy is $\mathrm{G}_{\mathrm{Epoxy}}$ $\sim 5 \mathrm{nW} / \mathrm{K}[9]$.

The detector can be operated in a constant-voltage bias mode [10] or an active bias mode [11]. The active bias mode is described in the next section. In the constant-voltage bias mode, the TES is voltage biased on the superconducting transition. Energy deposited by incident gamma-rays will thermalize in the absorber and TES, causing a small increase in the temperature and a large increase in the resistance of the TES. The increase in resistance causes the current through the TES to decrease, which is measured with a SQUID amplifier.

In the past, we have achieved an energy resolution of $70 \mathrm{eV}$ FWHM for $60 \mathrm{keV}$ gamma-rays using a constant-voltage biased TES with a $1 \mathrm{~mm} \times 1 \mathrm{~mm} \times 0.25 \mathrm{~mm}$ Sn absorber [8].

However, the count rate performance of our microcalorimeter was limited by a slow recovery time to less than $50 \mathrm{~Hz}$. Furthermore, a constant-voltage biased TES is sensitive to non-linearities in the superconducting transition which cause a non-linear detector response. These artifacts can arise from a variety of sources, such as contanination of the TES film, poor lithography during microfabrication, and stress exerted on the TES film by the epoxy.

\section{ACTIVE Negative FeEdBack Voltage Bias}

In the active bias mode, the bias voltage of the TES is actively reduced in proportion to the change in current of the TES during a pulse, such that the reduction in Joule power bias will maintain the TES at near-constant temperature. This 
allows the detector to recover very quickly. It also remedies the non-linear response due to non-linearities in the trarısition.

Active bias can be explained most easily by considering a pair of coupled, first-order, differential equations which describe our microcalorimeter geometry:

$$
\begin{aligned}
& C_{A b s} \frac{d T_{A b s}}{d t}=-\int_{T_{T E S}}^{T_{A b r}} G_{E p o x y} \cdot d T \\
& C_{T E S} \frac{d T_{T E S}}{d t}=\int_{T_{T E S}}^{T_{\text {Abs }}} G_{E p o x y} \cdot d T-\int_{T_{\text {suth }}}^{T_{\text {TEs }}} G_{S i N} \cdot d T+P_{\text {Bias }}
\end{aligned}
$$

where $C_{T E S,} C_{A b s}, T_{T E S}$, and $T_{A b s}$ is the respective heat capacity and temperature of the TES and absorber, and $G_{E p o x y}, G_{S i N}$ is the respective thermal conductivity of the epoxy and $\mathrm{SiN}$ membrane. The temperature of the cold bath is denoted by $T_{\text {Barh }}$. The operating bias power applied to the TES is $P_{\text {Bias }}$.

In the case of constant $P_{B i a s}$, the solution to the differential equations is a simple exponential. An absorbed gamma-ray with energy $E$ will increase the temperature of the TES by $\Delta T_{T E S}=E / C_{\text {Total }}$, where $C_{\text {Total }}=C_{\text {Abs }}+C_{T E S}$. The TES will return to equilibrium with a characteristic decay time constant of $\tau_{0}=C_{\text {Tota }} / G_{S i N}$

In the constant-voltage bias mode, the operating bias power is given by $P_{\text {Bias }}=V^{2} / R$, where $R$ is the resistance of the TES [10]. At equilibrium, the operating temperature of the TES is elevated above the bath temperature to transition by $P_{\text {Bias }} / G_{S i N}$. An absorbed gamma-ray increases the temperature and resistance of the TES. At the same time, $P_{B i a s}$ is intrinsically reduced, allowing the TES to retum to equilibrium faster. The solution of the differential equations has an effective time constant $\tau_{\text {Voltage-bias }}<\tau_{0}$, and a change in temperature of the TES $\Delta T_{T E S}<E / C_{\text {Total }}$.

In the active bias mode, the operating bias power is given by $P_{\text {Bias }}=I V$, with $I$ and $V$ related by:

$$
V=V_{O}+R_{\text {Gain }} \times\left(I-I_{O}\right) \text {, }
$$

where $V_{0}, I_{0}$ are the equilibrium voltage and current values, and $R_{\text {Gain }}$ is the feedback gain of a transimpedance amplifier [11]. During a pulse, the voltage bias applied to the TES is reduced in proportion to the change in current through the TES. The reduction in $P_{\text {Bias }}$ in this mode is greater thar in the constant-voltage bias mode. The solution of the differential equations has an effective time constant $\tau_{\text {Active-bias }}<\tau_{\text {vallage-bias }}$ $<\tau_{0}$, and change in temperature of the TES $\Delta T_{T E S}<<E / C_{\text {Total }}$.

For optimum operation of the active bias, it is essential that the energy flow rate from the absorber to the TES be smaller than $P_{\text {Btas }}$ at all times. Otherwise, the TES cannot not be maintained at constant temperature. In our devices, the energy flow from the absorber to the TES is limited by the thermal conductivity of the epoxy and its boundaries.

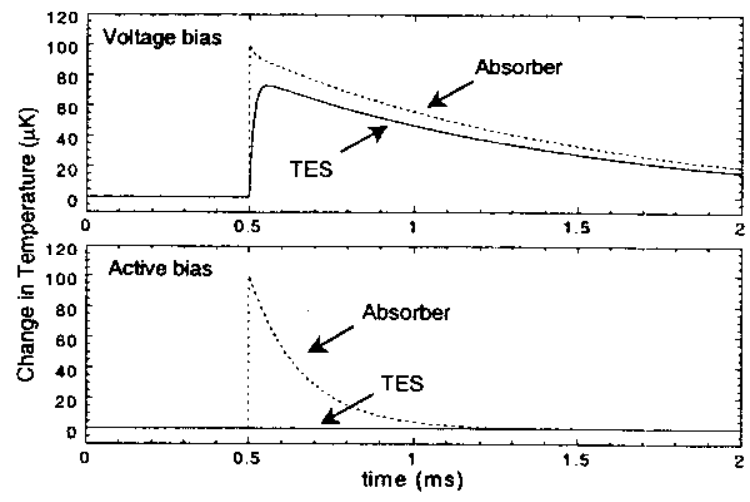

Fig. 1. Numerical model of two-element detector operating in voltage bias and active bias modes. Dotted lines represents change in temperature of the absorber. Solid lines represent change in temperature of the TES.

We have extensively modeled our detector operating in both bias modes. A temperature profile simulation is shown in fig 1 . In the voltage bias mode, both the absorber and TES heat up before the photon energy is dissipated into the cold bath. In the active bias mode, the reduction in $P_{B i a s}$ maintains the TES at near-constant temperature. The absorber cools off more quickly due to a larger temperature difference between the absorber and TES.

Active bias thus shortens the recovery time of the detector. Active bias also allows operation of the TES at near-constant temperature, making it impervious to any non-linearities, or "kinks", in the superconducting transition. A detector operating with an active bias will have higher count rates and a linear response.

\section{EXPERIMENTAL RESULTS}

\section{A. Pulse Shortening}

We have operated a detector with a constant voltage bias and an active bias. We measured pulses of different energies ranging from $60 \mathrm{keV}^{241} \mathrm{Am}$ gamma-rays, Sn escape lines, $\mathrm{Np}$ L-lines, down to $8 \mathrm{keV} \mathrm{Cu}$ fluorescence from the sample mount. Fig. 2 shows normalized typical current pulses measured in both bias modes. We observed a significant pulse shortening with the active bias by a factor of three. Our numerical model fits the measured pulses reasonably well. Discrepancies between the measurement and the model in the bottom part of the pulse tail are attributed to long thermalization times in the absorber. Efforts are underway to understand these features [9].

Because of the two-element design of the detector, the pulse shortening is limited by the time constant of energy flowing out of the absorber through the epoxy, given by $C_{A b d} / G_{\text {Epory }}$. So to further shorten the pulse, we would need either a smaller absorber heat capacity, or better thermal coupling between the absorber and TES. 


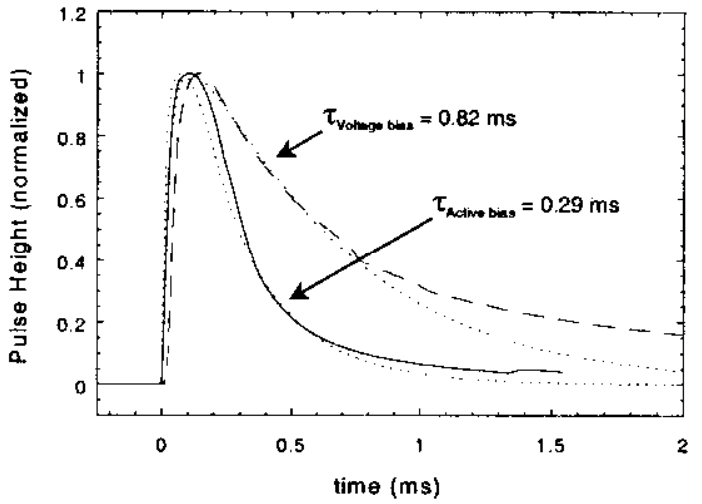

Fig. 2. Normalized current pulses showing pulse shortening. Dashed line is an averaged pulse taken with voltage bias, solid line is an averaged pulse taken with active bias. Dotted lines are numerical models for each respective case.

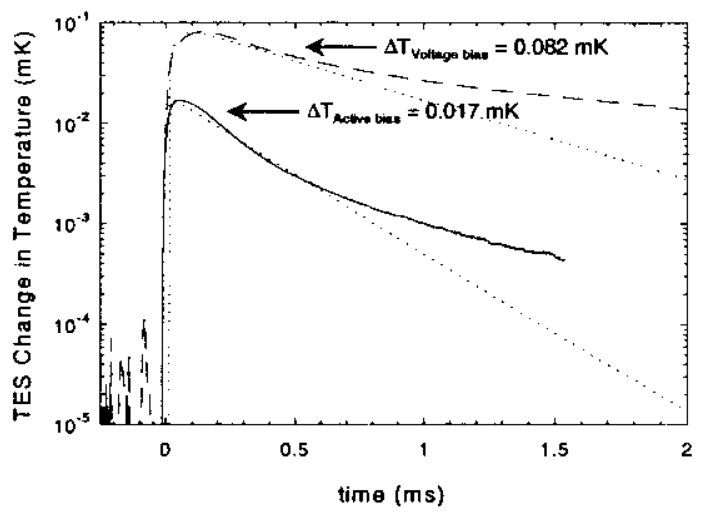

Fig. 3. Change in temperature of TES. Dashed line is an averaged pulse taken with voltage bias, solid line is an averaged pulse taken with active bias. Dotted lines are numerical models for each respective case.

\section{B. Linearization of Detector Response}

Knowledge of the superconducting transition [8] allows us to infer the change in temperature of the TES in both modes (fig. 3). We see that the change in TES temperature $\Delta T_{T E S}$ in the active bias mode is nearly an order of magnitude smaller than in the voltage bias mode, as expected. The reduction in the change in temperature of the TES during a pulse decreases the possibility of encountering non-linear artifacts in the transition.

The significant reduction in the change in temperature of the TES during a pulse linearizes the detector response (fig 4). We have taken data in the voltage bias mode, low-gain active bias mode, and high-gain active bias mode. The gain of the active bias can be adjusted by changing the value of $K_{\text {Gain }}$ in (2). In the low-gain active bias mode, all the effects of active bias are present but to a lesser degree than in the high-gain active bias mode.
With the voltage bias, the detector response is non-linear due to the non-linear transition of the TES. With the low-gain active bias, the low energy response is closer to linear, but the high energy response is still non-linear. With a high-gain active bias, the low energy response is completely linear. However, the high energy response remains non-linear. Analysis of the active biased pulse shape at $60 \mathrm{keV}$ reveals the cause (fig. 4 top inset). For high energy gamma-rays (>30 $\mathrm{keV}$ ), the energy rate flowing from the absorber into the TES is greater than the equilibrium bias power applied to the TES. The active bias is unable to reduce $P_{\text {Bias }}$ enough to compensate for the incoming energy. The reduction saturates, or rather "bottoms out", at zero power and cannot maintain the TES at near-constant temperature. In contrast, fig. 4 bottom inset shows a $60 \mathrm{keV}$ pulse taken with the voltage bias. The current pulse is not saturated, indicating that its non-linear response is an artifact of the transition. All pulses, saturated and unsaturated, agree with a single comprehensive numerical model whose only free parameters are incident photon energy and active bias gain.

\section{Energy Resolution Unchanged}

Fig. 5 shows the low energy spectra taken with voltage bias, low-gain active bias, and high-gain active bias. After compensating for non-linearity in the voltage bias case, all three spectra exhibit the same energy resolution of $120 \mathrm{eV}$ for $17.75 \mathrm{keV}$ x-rays. Note that significant peak shifting is noticeable for the $21 \mathrm{keV} x$-ray near the pulse height of 1.2 a.u., indicating the onset of non-linear behavior. The unchanged energy resolution ensures that, when detector design is optimized for active bias operation, there will be no degradation of resolving power.
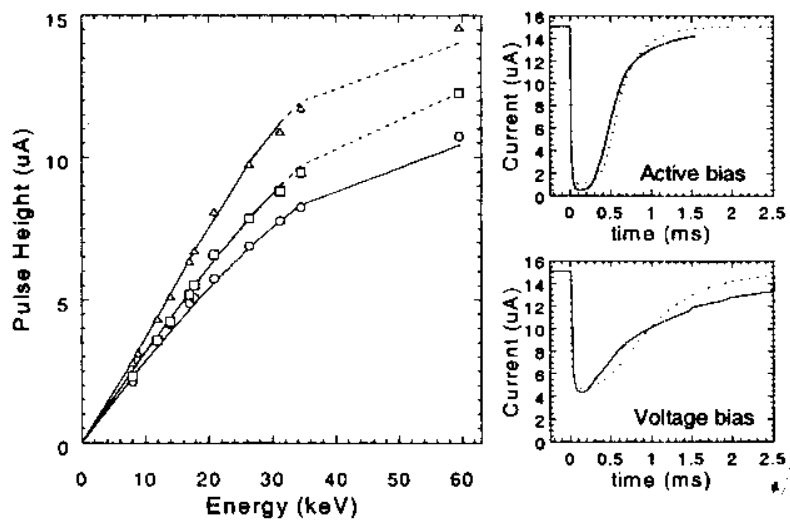

Fig. 4. Detector response as a function of energy for different modes of bias. Main figure: circles are measured pulse heights using voltage tias, squares are measured pulse heights using low-gain active bias, triangles are measured pulse heights using high-gain active bias. All lines are numerical models of each respective mode. Dotted lines indicate regions of pulse saturation. Insets: Solid lines are measured $60 \mathrm{keV}$ pulses in active bias and voltage bias modes. Dotted lines are numerical models of each respective mode. 


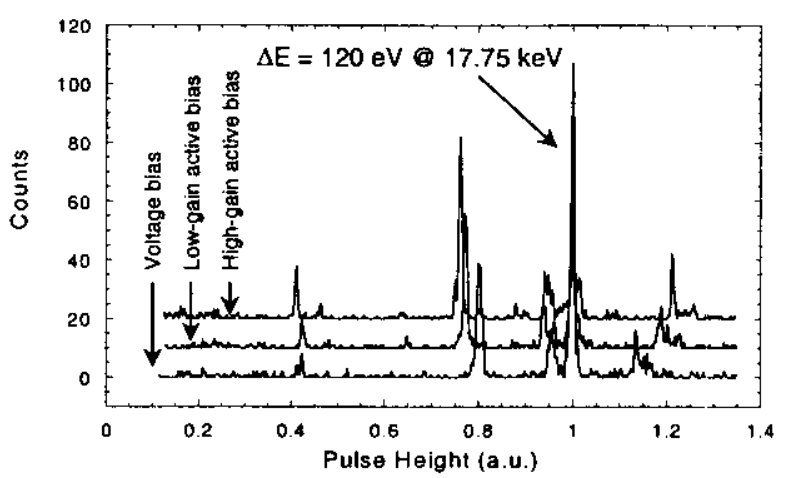

Fig. 5. Spectra taken in voltage bias, low-gain active bias, and high-gain active bias modes. After compensation for non-linearities, all three spectra exhibit similar resolution of $120 \mathrm{eV}$ for $17.75 \mathrm{keV} x$-rays.

\section{CONCLUSIONS}

We have demonstrated that active negative feedback voltage bias improves our high energy-resolution, high absorption-efficiency gamma-ray spectrometer. The improvements include speeding up detector recovery time to increase the count rate, and linearization of detector response by holding the TES at near-constant temperature during a pulse. More improvements can be made to maximize performance.

Currently, our pulse shortening is limited by the thermal conductivity of the epoxy. By increasing the contact area of the epoxy, we can further shorten the pulse length and increase the count rate.

In order to linearize the detector response over a large energy range, several improvements can be made. The most desirable of which is to increase $\boldsymbol{P}_{B \text { Bas }}$ by a combination of decreasing $T_{B a t h}$ and increasing $G_{S i N}$. The only criteria is that $G_{S i N}$ must be significantly smaller than $G_{E p o x y}$.

In the next iteration of this detection system, these improvements will be implemented. Results will no doubt be interesting and promising.

\section{ACKNOWLEDGMENT}

Many thanks to Jan Batteux for excellent experimental and technical support.

\section{REFERENCES}

[1] D. T. Chow, M. A. Lindeman, M. F. Cunningham, M. Frank, T. W. Barbee, Jr., S. E. Labov, "Gamma-ray spectrometers using a bulk $\mathrm{Sn}$ absorber coupled to a $\mathrm{Mc} / \mathrm{Cu}$ multiplayer superconducting transition edge sensor," $\mathrm{NuCl}$. Instr. Meth. A, vol. 444, pp. 196-200, 2000.

[2] S. H. Moseley, J. C. Mather, D. McCammon, "Thermal detectors as X-ray spectrometers," J. Appl. Phys., vol. 56(5), pp. $1257-1262,1984$.
[3] S. Vitale, G. Gallinaro, F. Gatti, "Alpha- beta- and gamma-ray detection with microcalorimeters made with superconducting absorbers," EUV, X-Ray, and Gamma-Ray Instrumentation for Astronomy $\mathrm{II}, \mathrm{O}$. H. W. Siegmund, ed., Proc. SPIE, vol. 1743, pp. 368-379, 1992.

[4] G. Forster, P. Ferger, F. von Feilitzsh, R. L. Mossbauer, "Calorimetric particle detectors with superconducting absorber materials," Nucl. Instr. Meth. A, vol. 324, pp. 491495, 1993.

[5] E. Silver, M. LeGros, G. Austin, N. Madden, J. Beeman, E. Haller, "First use of NTD germanium-based microcalorimeters for high-resolution broadband $x$-ray microanalysis," X-Ray Spectrom., vol. 25, pp. 265-268, 1997. [6] A. Alessandrello, J. W. Beeman, C. Brofferio, 0. Cremonesi, E. Fiorini, A. Giuliani, E. E. Haller, A. Monfardini, A. Nucciotti, M. Pavan, G. Pessina, E. Previtali, L. Zanotti, "High energy resolution bolometers for nuclear physics and x-ray spectroscopy," Phys. Rev. Lett., vol. 82, pp. 513-515, 1999.

[7] A. Bleile, P. Egelhof, H. J. Kluge, U. Liebisch, D. McCammon, H. J. Meier, O. Sebastian, C. K. Stahle, M. Weber, "Low-temperature x-ray detectors for precise Lamb shift measurements on hydrogen-like heavy ions," Nucl. Instr. Meth. A, vol. 444, pp. 488-491, 2000.

[8] D. T. Chow, A. Loshak, M. L. van den Berg, M. Frank, T. W. Barbee Jr., S. E. Labov, "High-resolution gamma-ray spectrometers using bulk absorbers coupled to $\mathrm{Mo} / \mathrm{Cu}$ multilayer superconducting transition edge sensors," to be published in Proc. SPIE, vol. 4141, 2000.

[9] M. L. van den Berg, D. T. Chow, A. Loshak, M. F. Cunningham, T. W. Barbee Jr., M. Frank, S. E. Labov, "High-resolution hard X-ray and gamma-ray spectrometers based on superconducting absorbers coupled to superconducting transition edge sensors," to be published in Proc. SPIE, vol. 4140, 2000.

[10] K. D. Irwin, G. C. Hilton, D. A. Wollman, J. M. Martinis, "X-ray detection using a superconducting transitionedge sensor microcalorimeter with electrothermal feedback," Appl. Phys. Lett., vol. 69, pp. 1945-1947, 1996.

[11] S. W. Nam, B. Cabrera, P. Colling, R. M. Clarke, E. Figueroa-Feliciano, A. J. Miller, R. W. Romani, "A new biasing technique for transition edge sensors with electrothermal feedback," IEEE Trans. On Appl. Supercond., vol. 9, pp. 4209-4212, 1999. 